\section{Dr. Rossides, et al reply}

To the Editor:

We thank Dr. Băicuş for his interest ${ }^{1}$ in our investigation of the mortality and functionality after stroke in individuals with systemic lupus erythematosus (SLE) ${ }^{2}$. In our article, we reported that the median age at stroke onset in individuals with SLE was 68 years for ischemic stroke and 65 for hemorrhagic stroke ${ }^{2}$. The mean time from SLE diagnosis to stroke onset was about 3 years for both stroke types, with great variability around the mean ${ }^{2}$. We stress that reporting the mean and SD might be useful for data description even in cases in which a variable is not normally distributed ${ }^{3}$.

Nonetheless, and given the opportunity of Dr. Băicuş's commentary, we have estimated the median age at SLE diagnosis; it was 59 years for ischemic stroke [interquartile range (IQR) 46-69 yrs] and 51 years for hemorrhagic stroke (IQR 40-63 yrs). It is important to distinguish between the age at SLE onset and the age at SLE diagnosis. The first we cannot readily identify with our data. The latter we can only approximate for some because the outpatient component of the National Patient Register became available in 2001, 3 years after study start. This means that for some of the patients included, their first diagnosis in our data was identified from the inpatient component only. Moreover, we should note that a higher age at SLE diagnosis is anticipated because our study population was restricted to individuals with SLE and stroke, and as we have shown in an earlier publication, stroke is rarer in individuals with SLE who are younger than 60 years ${ }^{4}$.

MARIOS ROSSIDES, MD, MSc, Karolinska Institutet, Clinical Epidemiology Division, Department of Medicine Solna, Stockholm, Sweden; JULIA F. SIMARD, ScD, Stanford School of Medicine, Health
Research and Policy, Division of Epidemiology, Stanford, California, USA, and Karolinska Institutet, Clinical Epidemiology Division, Department of Medicine Solna; ELISABET SVENUNGSSON, MD, PhD, Karolinska Institutet, Rheumatology Unit, Department of Medicine Solna and Karolinska University Hospital; MIA VON EULER, MD, PhD,

Karolinska Institutet, Stroke Research Network, Södersjukhuset, and Karolinska Institutet, Department of Clinical Science and Education; ELIZABETH V. ARKEMA, ScD, Karolinska Institutet, Clinical Epidemiology Division, Department of Medicine Solna, Stockholm, Sweden. Address correspondence to Dr. M. Rossides, Karolinska Institutet, Department of Medicine Solna, Clinical Epidemiology Division, T2, 17176 Stockholm, Sweden. E-mail: marios.rossides@ki.se

\section{REFERENCES}

1. Băicuş C. At what age was SLE diagnosed in this cohort? J Rheumatol 2018;45:1070.

2. Rossides M, Simard JF, Svenungsson E, von Euler M, Arkema EV. Mortality and functionality after stroke in patients with systemic lupus erythematosus. J Rheumatol 2017;44:1590-6.

3. Lydersen S. Statistical review: frequently given comments. Ann Rheum Dis 2015;74:323-5.

4. Arkema EV, Svenungsson E, von Euler M, Sjowall C, Simard JF. Stroke in systemic lupus erythematosus: a Swedish population-based cohort study. Ann Rheum Dis 2017;76:1544-9.

J Rheumatol 2018;45:7; doi:10.3899/jrheum.180122 